\title{
Severe Osteoporosis in a Young Woman With Celiac Diseases due to Vitamin D Deficiency
}

\author{
Merita Emini $^{\mathrm{a}}$, Nadije Morina ${ }^{\mathrm{a}, \mathrm{d}}$, Idriz Gerqari ${ }^{\mathrm{b}}$, Ilir Alimehmetic ${ }^{\mathrm{c}}$
}

\begin{abstract}
Osteoporosis and other related clinical features from secondary hyperparathyroidism due to the intestinal malabsorption of vitamin D and calcium can often be misdiagnosed. Symptoms such as weight loss, gastrointestinal discomfort, hypocalcemia, anemia and neurological symptoms like paresthesia and leg pain have to be considered as celiac disease (CD)-related consequences. We report the case of a 37-yearold woman with biannual history of weight loss, extreme fatigue, chest pain, paresthesia, and leg pain. After full biochemical and endocrine assessment, we found anemia with severe iron deficiency, hypocalcemia, vitamin D deficiency and high parathyroid hormone level. Moreover, on whole body dual-energy X-ray absorptiometry, osteoporosis with diffuse metabolic changes was present, antibodies for $\mathrm{CD}$ were positive and renal function was normal. After these examinations, the patient was diagnosed with secondary hyperparathyroidism due to CD. Treatment with gluten-free diet, high doses of vitamin D and calcium revealed osteoporosis and hyperparathyroidism was treated for several months. Secondary hyperparathyroidism due to $\mathrm{CD}$ has to be considered during the evaluation of patients with weight loss, hypocalcemia, anemia and other non-specific metabolic, neurological and gastrointestinal symptoms. Early gluten-free diet treatment, high doses of vitamin D supplements and calcium must be used to treat osteoporosis and secondary hyperparathyroidism in the meantime. Treatment with bisphosphonates is not necessary for osteoporosis due to $\mathrm{CD}$.
\end{abstract}

Keywords: Vitamin D deficiency; Hyperparathyroidism; Celiac disease

\section{Introduction}

Celiac disease (CD) is an autoimmune disorder caused by glu-

Manuscript accepted for publication August 10, 2016

${ }^{a}$ Clinic of Endocrinology, University Clinical Centre of Kosova, Prishtine, Republic of Kosovo

bInstitute of Nuclear Medicine, University Clinical Centre of Kosova, Prishtine, Republic of Kosovo

'Infectious Diseases and Occupational Health Department, Faculty of Medicine, University of Medicine, Albania

${ }^{\mathrm{d}}$ Corresponding Author: Nadije Morina, Clinic of Endocrinology, University Clinical Centre of Kosova, Prishtine, Republic of Kosovo.

Email: nadije78@hotmail.com

doi: http://dx.doi.org/10.14740/jmc2601w ten in the diet of genetically predisposed individuals and is considered as a major cause of intestinal malabsorption. Villous atrophy in CD that leads to calcium and vitamin D malabsorption that results in reduction of calcium intake and secondary hyperparathyroidism are the main reasons for the increased rate of osteoporosis and risk for fractures previously reported $[1,2]$. Prevalence of such disease is approximately $1 \%$ worldwide $[3,4]$. Besides gluten-free diet (GFD), which is the main approach in these patients, vitamin $\mathrm{D}$, calcium and bisphosphonates are the treatment of choice. We report here the case of a 37-year-old woman with biannual history of extreme fatigue, chest pain, paresthesia, leg pain and severe weight loss during the last 12 months. Whole body bone scintigraphy revealed severe osteoporosis and extreme hyperparathyroidism with low calcemia. CD was diagnosed by means of positive serologic tests for antibodies and was confirmed through histopathological examination.

\section{Case Report}

A 37-year-old woman presented with weight loss, low appetite, and joint pain mainly localized in knees, ankles, lower back and chest. She was hospitalized in the neurology department for pain and paresthesia of the low extremities. The patient presented normal physical and mental development. During that period, she underwent osteodensitometry that revealed severe osteoporosis with diffuse focal fields in the joints, after which she was asked for parathyroid hormone level (PTH) examination that was found to be very high, $351.8 \mathrm{pg} / \mathrm{mL}$ (normal values $12.0-72.0 \mathrm{pg} / \mathrm{mL}$ ) and high alkaline phosphatase, $291 \mathrm{U} / \mathrm{L}$ (normal values 37 - $98 \mathrm{UI} / \mathrm{L}$ for her age). She presented with hypocalcemia and iron deficiency anemia unresponsive to oral iron therapy. We were asked for an endocrinologist consultation and she was transferred immediately in the Clinic of Endocrinology for further examination and treatment with the diagnosis of suspected hyperthyroidism secondary to $\mathrm{CD}$.

She presented no previous history of metabolic disease, her parents were alive, and she was married and had three healthy children. During 2 years, she was taking analgetics, when she had hard pain. She was living with her family and was unemployed. During the last year, she complained about difficulties to walk, morning stiffness, poor energy, pain in the chest and/or lower back especially during the supine rest, and difficulty standing up for a long time. She had abdominal pain and nausea and sometimes experienced vomiting after eating. She presented a weight loss of $10 \mathrm{~kg}$ in 12 months. During 
Table 1. Biochemical Analysis Before and After Treatment

\begin{tabular}{llllll}
\hline & On admission & After $\mathbf{4}$ months & After $\mathbf{7}$ months & After $\mathbf{1 0}$ months & Reference value \\
\hline 25-OH-vitamin D, ng/mL & 3 & 24.8 & 39.3 & 34.8 & $\begin{array}{l}25.0-100 \text { normal } \\
10.0-24.9 \text { deficiency } \\
\end{array}$ \\
& & & & $<9.9$ insufficiency \\
PTH, pg/mL & 407 & 263 & 86.9 & 36.8 & $12.0-72.0$ \\
Total calcium, mmol/L & 1.58 & 2.18 & NA & 2.19 & $2.15-2.57$ \\
Ionized calcium, mmol/L & 1.05 & 1.13 & $\mathrm{NA}$ & 1.15 & $1.12-1.32$ \\
\hline
\end{tabular}

PTH: parathyroid hormone.

the physical examination, she was found to present demineralization of her teeth which she had artificially replaced. She presented no regional lymphadenopathy, no joints deformity, proximal muscle weakness and generalized muscle atrophy. Chvostek's and Trousseau's signs were positive. No pathologic findings were present in the cardiovascular and respiratory systems and painful epigastrium on deep palpation was found. She was a non-smoker and did not consume alcohol.

\section{Investigations and diagnosis}

Laboratory analyses revealed high PTH, low calcium and $25(\mathrm{OH})$ vitamin $\mathrm{D}$ (Table 1 ) and anemia with low iron value (Table 2).

Liver function tests were within the normal range as were also renal specific analyses. Inflammatory markers were negative. Thyroid function tests were normal. Thoracic magnetic resonance imaging (MRI) showed degenerative bone changes. Chest X-ray was normal. Ultrasound of abdomen showed normal liver, gallbladder, bile ducts, pancreatic head and neck. A dual-energy X-ray absorptiometry (DXA) scan showed marked changes which were interpreted as indicative of low bone mass for her age and severe osteoporosis (Table 3).

In the meantime, whole body bone scintigraphy revealed diffuse osteoporosis characteristic for metabolic bone disease (Fig. 1). Celiac serology showed positive anti-gliadin (20.1 $\mathrm{AU} / \mathrm{mL}$ ) and negative anti-tissue transglutaminase (5.3 AU/ $\mathrm{mL}$ ). Thyroid functional tests were within normal range. Serum Helicobacter pylori and brucellosis tests (ASHB) were negative. An oesophagogastroduodenoscopy showed mild sub mucosal lesion. On histology, total resorptive villous depletion and intensive mononuclear inflammatory infiltration were revealed. Colonoscopy was also performed and internal grade II hemorrhoid nodules were found. She presented sinusal rhythm, 60 beat per minute cardiac frequency and no pathologic signs were found on electrocardiogram (ECG). Thyroid and neck ultrasound was performed revealing an isoecogenic nodule with hypoecogenic limits, diameter of $10 \mathrm{~mm}$, in the low pole posterior of the right thyroid lobe, which remained same even after the treatment. CD was diagnosed through serologic testes, based on the World Health Organisation (WHO) criteria [5] for severe osteoporosis due to secondary hyperthyroidism and severe concomitant iron deficiency anemia.

\section{Treatment}

GFD was immediately ordered and intramuscular cholecalciferol and intravenous calcium gluconate were administered, in order to replace vitamin D stores in the body. Moreover, intravenous medications for anemia were given. Oral daily vitamin D was continued after relieving gastrointestinal symptoms through GFD, approximately 50,000 UI per week in the beginning and after based on vitamin D serum value. Treatment with bisphosphonate was not necessary.

\section{Outcome and follow-up}

The patient was followed up monthly, for 3 months, during which period she showed marked clinical and biochemical im-

Table 2. Hemogram Before and After the Treatment

\begin{tabular}{llll}
\hline & On admission & After 10 months & Reference value \\
\hline Erythrocytes, /L & 3.3 & $4.6 \times 10^{-6}$ & $3.5-5.1 \times 10^{12}$ \\
\hline Hemoglobin, g/L & 94 & 128 & $110.0-150.0$ \\
Hematocrit, \% & 35.2 & 38 & $35.0-47.0$ \\
MCV, fL & 73 & 82 & $80.0-99.0$ \\
MCH, pg & 21.9 & 27.4 & $26.0-32.0$ \\
MCHC, g/L & 301 & 333 & $310.0-360.0$ \\
Iron, $\mu \mathrm{mol} / \mathrm{L}$ & 4.2 & 14.3 & $9.0-30.4$ \\
\hline
\end{tabular}

MCV: mean corpuscular volume; $\mathrm{MCH}$ : mean corpuscular hemoglobin; $\mathrm{MCHC}$ : mean corpuscular hemoglobin concentration. 
Table 3. DXA Scan Results Before and After the Treatment

\begin{tabular}{llllllll}
\hline \multirow{2}{*}{ Region } & \multicolumn{3}{c}{ On admission } & & \multicolumn{3}{c}{ After 8 months } \\
\cline { 2 - 3 } \cline { 7 - 8 } & BMD & T- & Z- & & BMD & T- & Z- \\
\hline Right femur & 0.371 & -4.7 & -4.6 & & 0.714 & -1.9 & -1.8 \\
Left femur & 0.375 & -4.6 & -4.5 & & 0.693 & -2.0 & -1.9 \\
L-spine & 0.461 & -5.3 & -5.2 & & 0.737 & -2.8 & -2.7 \\
\hline
\end{tabular}

BMD: bone mineral density.

provement. After 3 months, she gained $7 \mathrm{~kg}$. She did not experience paresthesia or joint pain and she felt better in general.

On follow-up analyses, vitamin D, PTH and calcium were satisfactory improved (Table 1), DXA scan revealed significant improvement from marked osteoporosis to osteopenia (Table 3) and full blood count also presented significant improvement (Table 2 ).

\section{Discussion}

We described the case of a young woman with osteoporosis and clinical, biochemical and imagery specific examinations.

Except in chronic renal failure, secondary hyperparathy- roidism can be due to $\mathrm{CD}$ related to vitamin $\mathrm{D}$ and calcium malabsorption, which can increase bone turnover.

In $C D$, there is a loss of callous surface area [6] with unabsorbed calcium binding to excess fatty acids in the intestinal lumen as a result of fat malabsorption [7], causing a negative calcium balance. Malabsorption occurred due to the loss of absorptive area from autoimmune process caused from gliadin. Hypocalcemia in the meantime stimulates PTH to correct this imbalance. People at risk of developing this disease carry alleles encoding HLA-DQ2 or DQ8, leading to an autoimmune reaction that is characteristic of the early stages of $\mathrm{CD}$ [8].

Patients with CD present nutritional deficiencies, most commonly iron, vitamin B12, vitamin B6, vitamin D, folate, and zinc deficiencies [9]. Neurologic disorders have already been reported in association with malabsorption of vitamin B12, folate, copper, and vitamin D [10].

Patients with CD should be evaluated for common concomitant autoimmune conditions, such as thyroid and liver diseases, as well as deficiencies in iron, vitamin D, and vitamin B12.

The main treatment is GFD, which alone has been shown to cause significant improvement in bone mineral density (BMD) and showed results similar to those achieved by bisphosphonates in the treatment of post-menopausal osteoporosis, with a reduction in bone remodeling [11, 12]. GFD is

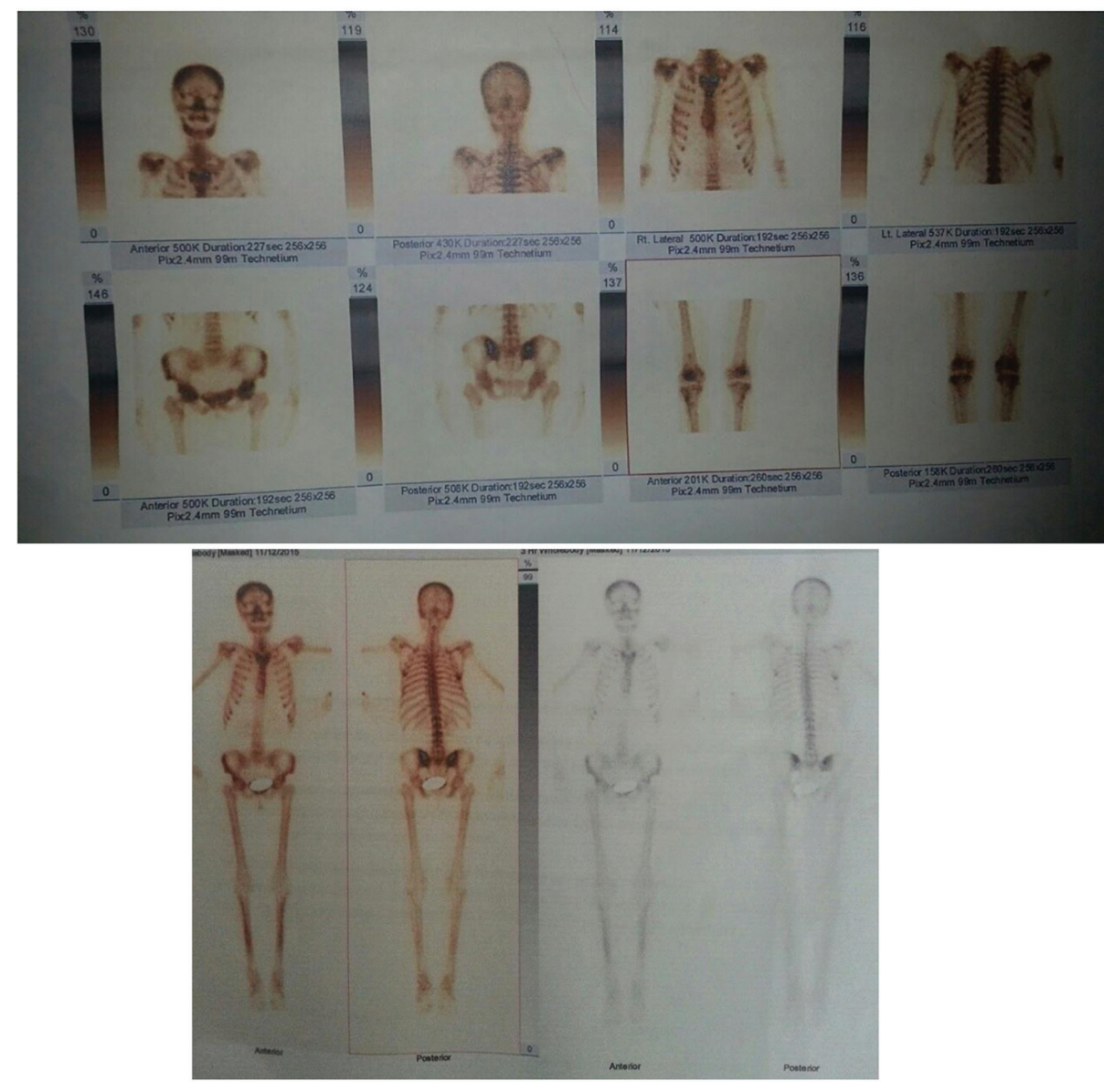

Figure 1. Whole body scintigraphy of bone in admission and after the treatment. 
also associated with reduction in PTH levels, improvement in vitamin $\mathrm{D}$ levels and other biochemical parameters in $\mathrm{CD}$ patients $[13,14]$.

In our patient with $\mathrm{CD}$ and secondary hyperparathyroidism, there was a significant improvement in DXA scan, clinical, biochemical parameters after 10 months of GFD, calcium and vitamin D supplementation. The significant improvement in BMD within a short time in our patient could be attributed to her relatively young age, early diagnosis of CD, proper compliance, adherence to GFD and adequate supplementation with vitamin $\mathrm{D}$ and calcium.

It is recommended that patients with $\mathrm{CD}$ should be examined at least twice in their first year after diagnosis in order to monitor symptoms, serologic features, nutrition, dietary adherence and body mass index [15-17]; meanwhile DXA scan evaluation for BMD is recommended during the first year after diagnosis [16].

In the meantime, bone loss relationship with PTH gives us important information for monitoring the treatment of CD, hence identifying those patients at particular risk of osteoporosis.

Estimation of PTH is crucial when facilities for the measurement of BMD are not uniformly available.

\section{Conclusion}

Secondary hyperparathyroidism due to CD has to be considered during the evaluation of patients with weight loss, hypocalcemia, anemia and non-specific metabolic, neurologic and gastrointestinal symptoms.

Early treatment with GFD, vitamin D and calcium cures osteoporosis and secondary hyperparathyroidism in the meantime.

Treatment with bisphosphonates is not necessarily needed in the treatment of osteoporosis from CD.

For populations with high prevalence for vitamin D deficiency or osteomalacia, biochemical evaluation for suspicious cases should include measurement of PTH, calcium, vitamin D, whole blood count and specific antibodies for CD, in order for such cases not to be overlooked and remain undiagnosed.

\section{Conflicts of Interest}

We declare no conflicts of interest in this case report.

\section{References}

1. Walters JR, Banks LM, Butcher GP, Fowler CR. Detection of low bone mineral density by dual energy $\mathrm{x}$ ray absorptiometry in unsuspected suboptimally treated coeliac disease. Gut. 1995;37(2):220-224.

2. Vasquez H, Mazure R, Gonzalez D, Flores D, Pedreira S, Niveloni S, Smecuol E, et al. Risk of fractures in celiac disease patients: a cross-sectional, case-control study. Am J Gastroenterol. 2000;95(1):183-189.
3. Rostom A, Murray JA, Kagnoff MF. American Gastroenterological Association (AGA) Institute technical review on the diagnosis and management of celiac disease. Gastroenterology. 2006;131(6):1981-2002.

4. Green PH, Cellier C. Celiac disease. N Engl J Med. 2007;357(17):1731-1743.

5. Assessment of fracture risk and its application to screening for postmenopausal osteoporosis. Report of a WHO Study Group. World Health Organ Tech Rep Ser. 1994;843:1-129.

6. Melvin KE, Hepner GW, Bordier P, Neale G, Joplin GF. Calcium metabolism and bone pathology in adult coeliac disease. Q J Med. 1970;39(153):83-113.

7. Harris OD, Philip HM, Cooke WT, Pover WF. 47Ca studies in adult coeliac disease and other gastrointestinal conditions with particular reference to osteomalacia. Scand J Gastroenterol. 1970;5(3):169-175.

8. Schuppan D. Current concepts of celiac disease pathogenesis. Gastroenterology. 2000;119(1):234-242.

9. Wierdsma NJ, van Bokhorst-de van der Schueren MA, Berkenpas M, Mulder CJ, van Bodegraven AA. Vitamin and mineral deficiencies are highly prevalent in newly diagnosed celiac disease patients. Nutrients. 2013;5(10):3975-3992.

10. McKeon A, Lennon VA, Pittock SJ, Kryzer TJ, Murray J. The neurologic significance of celiac disease biomarkers. Neurology. 2014;83(20):1789-1796.

11. Valdimarsson T, Lofman O, Toss G, Strom M. Reversal of osteopenia with diet in adult celiac disease. Gut. 1996;38:323-327.

12. Mautalen C, Gonzalez D, Mazure R, Vazquez H, Lorenzetti MP, Maurino E, Niveloni S, et al. Effect of treatment on bone mass, mineral metabolism, and body composition in untreated celiac disease patients. Am J Gastroenterol. 1997;92(2):313-318.

13. Kemppainen T, Kroger H, Janatuinen E, Arnala I, Lamberg-Allardt C, Karkkainen M, Kosma VM, et al. Bone recovery after a gluten-free diet: a 5-year follow-up study. Bone. 1999;25(3):355-360.

14. Sategna-Guidetti C, Grosso SB, Grosso S, Mengozzi G, Aimo G, Zaccaria T, Di Stefano M, et al. The effects of 1-year gluten withdrawal on bone mass, bone metabolism and nutritional status in newly-diagnosed adult coeliac disease patients. Aliment Pharmacol Ther. 2000;14(1):3543.

15. Bai JC, Fried M, Corazza GR, Schuppan D, Farthing M, Catassi C, Greco L, et al. World Gastroenterology Organisation global guidelines on celiac disease. J Clin Gastroenterol. 2013;47(2):121-126.

16. Rubio-Tapia A, Hill ID, Kelly CP, Calderwood AH, Murray JA. ACG clinical guidelines: diagnosis and management of celiac disease. Am J Gastroenterol. 2013;108(5):656-676; quiz 677.

17. Husby S, Koletzko S, Korponay-Szabo IR, Mearin ML, Phillips A, Shamir R, Troncone R, et al. European Society for Pediatric Gastroenterology, Hepatology, and Nutrition guidelines for the diagnosis of coeliac disease. J Pediatr Gastroenterol Nutr. 2012;54(1):136-160. 